\section{A Computational Method for Simultaneous LQ Optimal Control Design via Piecewise Constant Output Feedback}

\author{
Yong-Yan Cao and James Lam
}

\begin{abstract}
This paper is concerned with simultaneous linear-quadratic (LQ) optimal control design for a set of LTI systems via piecewise constant output feedback. First, the discrete-time simultaneous LQ optimal control design problem is reduced to solving a set of coupled matrix inequalities and an iterative LMI algorithm is presented to compute the feedback gain. Then, simultaneous stabilization and simultaneous LQ optimal control design of a set of LTI continuous-time systems are considered via periodic piecewise constant feedback gain. It is shown that the design of a periodic piecewise constant feedback gain simultaneously minimizing a set of given continuous-time performance indexes can be reduced to that of a constant feedback gain minimizing a set of equivalent discrete-time performance indexes. Explicit formulas for computing the equivalent discrete-time systems and performance indexes are derived. Examples are used to demonstrate the effectiveness of the proposed method.
\end{abstract}

Index Terms-Optimal control, periodic systems, piecewise constant output feedback, simultaneous stabilization.

\section{INTRODUCTION}

Simultaneous stabilization of a collection of plants is an important problem in the area of robust control design. It is concerned with the determination of a single controller which simultaneously stabilize a finite collection of plants. The technique finds its applications in stabilizing linear plants characterized by different modes of operation (for instance, failure modes) or nonlinear plants linearized at several equilibria. In designing a control system with integrity, we have a nominal model, say $P_{0}$, and at some point a structural change, such as a loss of a sensor or actuator, may occur, yielding a new model in the finite set $\left\{P_{1}, \ldots, P_{r}\right\}$. In general, we expect to design a single controller which not only stabilizes every possible model in the finite set, but also provides acceptable performance.

Recently, Paskota et al. [12] proposed a computational technique for optimal simultaneous stabilization for linear single-input systems via linear state feedback control. In [4], an iterative LMI algorithm was proposed to obtain output/state feedback gain for a collection of multiple-input multiple-output (MIMO) continuous-time plants. Cao et al. [5] proved that a finite number of continuous-time MIMO plants are simultaneously stabilizable via state feedback and static output feedback if and only if a set of coupled linear-quadratic (LQ) control problems with some compatible cross terms in the cost functional is feasible and then it is reduced to a coupled algebraic matrix inequalities (ARIs) problem. Lam and Cao [11] addressed the LQ simultaneous optimal control design for a set of continuous-time systems using the LMI-based approach.

A multirate approach is presented in Khargonekar et al. [9], where a simultaneously stabilizing control law based on periodic dynamic compensators is proposed. The basic idea is to divide an output sampling interval into as many subintervals as there are plants to be stabilized and to implement a deadbeat controller for each plant. Although the

Manuscript received January 17, 2001; revised April 24, 2001. This work was supported in part by Hong Kong University CRCG Grant 10203013/19870. This paper was recommended by Associate Editor Y. Lee.

Y.-Y. Cao is with the Department of Electrical and Computer Engineering, University of Virginia, Charlottesville, VA 22903 USA (e-mail: yycao@virginia.edu).

J. Lam is with the Department of Mechanical Engineering, The University of Hong Kong, Hong Kong, China (e-mail: jlam@ @ku.hk).

Publisher Item Identifier S 1083-4419(01)08454-0. approach employed is quite general, an issue it does not address is the closed-loop performance. This is because excessive intersampling ripple may occur under the restriction of deadbeat control. As a consequence, the closed-loop system may exhibit large overshoots and may be sensitive to parameters in many cases [6]. Although the problem of constant output feedback stabilization is still open since the nonconvexity of the solution set, which renders it a nontrivial computational task, analytical and numerical alike. Fortunately, it was shown in [2] that, if a system is controllable and observable, then for almost all output sampling rates, any self-conjugate pole configuration can be assigned to the discretized closed-loop system by periodic piecewise constant output feedback, provided that the number of gain changes is not less than the system controllability index. As a consequence, such a control law can stabilize a much larger class of systems than a constant output feedback.

In this paper, the simultaneous optimal control design is first addressed using the LMI approach for a collection of discrete-time MIMO plants via constant output feedback control. We show that the design of optimal simultaneous stabilization controller for a collection of discrete-time systems can be reduced to an optimization problem subject to a set of coupled matrix inequalities. Simultaneously optimal design of a set of LTI systems using periodic piecewise constant output feedback is then addressed. We show that the problem can be reduced to the design of an constant output feedback gain minimizing a set of equivalent discrete-time performance indexes for a set of LTI discrete-time systems. The explicit computing formulation of the equivalent discrete-time systems and performance indexes are derived.

\section{Simultaneous LQ Optimal Control For DISCRETE-TIME SYSTEMS}

Consider the following $r$ discrete-time systems:

$$
\begin{aligned}
x_{i}(k+1) & =A_{i} x_{i}(k)+B_{i} u_{i}(k) \\
y_{i}(k) & =C_{i} x_{i}(k)
\end{aligned}
$$

where
$x_{i} \in \mathcal{R}^{n_{i}}$
$u_{i} \in \mathcal{R}^{m}$
state vector;
$y_{i} \in \mathcal{R}^{p}$ control vector; control output vector for $i=1, \ldots, r$.

The matrices $A_{i}, B_{i}$, and $C_{i}$ are constant and with appropriate dimensions and the triple $\left(A_{i}, B_{i}, C_{i}\right)$ are assumed to be stabilizable and detectable. The problem considered in this section is the design of a fixed constant output feedback control law

$$
u_{i}(k)=F y_{i}(k)
$$

minimizing an upper-bound on the performance measures

$$
J_{i}=\mathcal{E}\left\{\sum_{k=0}^{\infty}\left[\begin{array}{l}
x_{i}(k) \\
u_{i}(k)
\end{array}\right]^{\mathrm{T}}\left[\begin{array}{ll}
Q_{i} & S_{i} \\
S_{i}^{\mathrm{T}} & R_{i}
\end{array}\right]\left[\begin{array}{l}
x_{i}(k) \\
u_{i}(k)
\end{array}\right]\right\}
$$

for $i=1, \ldots, r$, each associated with one of the plants (1), where standard linear-quadratic assumptions are made for each system, namely, all $Q_{i} \geq 0, R_{i}>0$, and $Q_{i}-S_{i} R_{i}^{-1} S_{i}^{\mathrm{T}} \geq 0$, and the mathematical expectation $\mathcal{E}$ is used to take into account the dependence of $J_{i}$ on the initial conditions. We assume that $x_{i}(0)$ is a random variable with zero mean and unit covariance.

It is well known that for a given $F$, the performance measures in (3) are given by

$$
J_{i}=\operatorname{trace}\left(P_{i}\right)
$$


where each $P_{i} \geq 0$ satisfies, the Lyapunov equation

$$
\bar{A}_{i}^{\mathrm{T}} P_{i} \bar{A}_{i}+\bar{Q}_{i}+\left(F C_{i}\right)^{\mathrm{T}} R_{i} F C_{i}=P_{i}, \quad i=1, \ldots, r
$$

where $\bar{A}_{i}=A_{i}+B_{i} F C_{i}, \bar{Q}_{i}=Q_{i}+S_{i} F C_{i}+\left(S_{i} F C_{i}\right)^{\mathrm{T}}$.

Lemma 1: Consider discrete-time systems (1) with compatible weighting matrices $\left(Q_{i}, R_{i}, S_{i}\right)$ for $i=1, \ldots, r$. The $i$ th system is stabilizable via constant output feedback if and only if there exist matrices $F$ and $G_{p i}$ such that

$$
\bar{R}_{i}^{1 / 2} F C_{i}+\bar{R}_{i}^{-1 / 2}\left(B_{i}^{\mathrm{T}} P_{i} A_{i}+S_{i}^{\mathrm{T}}\right)=G_{p i}
$$

where $\bar{R}_{i}=R_{i}+B_{i}^{\mathrm{T}} P_{i} B_{i}, P_{i} \geq 0$ is the solution of the algebraic Riccati equation

$$
\begin{gathered}
A_{i}^{\mathrm{T}} P_{i} A_{i}-\left(A_{i}^{\mathrm{T}} P_{i} B_{i}+S_{i}\right) \bar{R}_{i}^{-1}\left(B_{i}^{\mathrm{T}} P_{i} A_{i}+S_{i}^{\mathrm{T}}\right) \\
+Q_{i}+G_{p i}^{\mathrm{T}} G_{p i}=P_{i} .
\end{gathered}
$$

In this case, the performance index takes value as $J_{i}=\operatorname{trace}\left(P_{i}\right)$. If there exist real matrices $F$ and $G_{p i}$ such that the Riccati equation (6) have solutions $P_{i} \geq 0$ for $i=1, \ldots, r$, then the feedback gain simultaneously stabilizes the $r$ systems and minimizes the performance indexes (3) for $i=1, \ldots, r$.

Proof: Sufficiency: Substituting (5) into (6), it is easy to find that the Riccati equation (6) is equivalent to Lyapunov equation (4). Obviously, if there exist such matrices $F$ and $G_{p i}$, then Lyapunov equation will hold. This means that $F$ is a stabilizing feedback control law and minimizes the performance indexes (3). Necessity: Assume that there exists a feedback gain $F$ stabilizing every system, then Lyapunov equation (4) will have a solution $P_{i} \geq 0$. This means there exist real matrices $F$ and $G_{p i}$ satisfying (5) such that (6) has a solution $P_{i} \geq 0$.

Theorem 1 gives a necessary and sufficient condition for static output feedback stabilizability for a single discrete-time system. When $G_{p i}=$ 0 , (6) is the standard Riccati equation to construct a state feedback control law that minimizes the performance index (3). On the other hand, if Riccati equation (6) has a solution $P_{i} \geq 0$ with $G_{p i}=0$ and $F$ can be selected to satisfy constraint (5), from optimal control theory it is the optimal feedback gain via state feedback. Theorem 1 is an extension of the result in [10] for discrete-time systems, where a similar necessary and sufficient condition on the static output feedback stabilizability was established for the continuous-time systems with $Q_{i}=C_{i}^{\mathrm{T}} C_{i}, S_{i}=0$ and $R_{i}=I$. Since the concepts such as stabilizability and detectability are not explicitly employed in Theorem 1, the proof is comparatively much more transparent.

For a constant feedback gain $F$, which simultaneously stabilizes the $r$ systems, it generally does not minimize every performance index (3) of the corresponding single plant, but there exist $P_{i} \geq 0$ such that the following Lyapunov inequalities hold:

$$
\bar{A}_{i}^{\mathrm{T}} P_{i} \bar{A}_{i}+\bar{Q}_{i}+\left(F C_{i}\right)^{\mathrm{T}} R_{i} F C_{i}<P_{i}
$$

Generally, the performance values $\operatorname{trace}\left(P_{i}\right) \geq \operatorname{trace}\left(P_{i}^{0}\right)$ for $i=$ $1, \ldots, r$, where $P_{i}^{0} \geq 0$ is the solution of Lyapunov equation (4). Therefore, we aim to determine an output feedback gain to minimize the sum of the performance index (3), i.e.,

$$
J=\sum_{i=1}^{r} \operatorname{trace}\left(P_{i}\right)
$$

where $P_{i} \geq 0$ satisfies Lyapunov inequality (7) for $i=1, \ldots, r$.

\section{ILMI APPROACH to SimUltANEOUS OPTIMAL DESIGN}

Note that for any $P_{i}^{0} \geq 0$ satisfying Lyapunov equation (4), $P_{i}>$ $P_{i}^{0}$ holds for any $P_{i}>0$ satisfying Lyapunov matrix inequality (7) [3]. It is equivalent to

$$
\left[\begin{array}{cc}
\bar{Q}_{i}+\left(F C_{i}\right)^{\mathrm{T}} R_{i} F C_{i}-P_{i} & \left(A_{i}+B_{i} F C_{i}\right)^{\mathrm{T}} P_{i} \\
P_{i}\left(A_{i}+B_{i} F C_{i}\right) & -P_{i}
\end{array}\right]<0
$$

which may be rewritten as

$$
\left[\begin{array}{cc}
\bar{Q}_{i}-P_{i} & A_{i}^{\mathrm{T}} P_{i} \\
P_{i} A_{i} & -P_{i}-P_{i} B_{i} R_{i}^{-1} B_{i}^{\mathrm{T}} P_{i}
\end{array}\right]+\Xi_{i}^{\mathrm{T}} \Xi_{i}<0
$$

where $\Xi_{i}=\left[\begin{array}{lll}R_{i}^{1 / 2} F C_{i} & R_{i}^{-1 / 2} B_{i}^{\mathrm{T}} P_{i}\end{array}\right]$.

Remark 1: It is clear that (7) with $Q_{i}=0, S_{i}=0$, and $R_{i}=I$ is a necessary and sufficient condition for the stabilizability via a constant output feedback for a single LTI discrete-time system.

Inequality (9) is still a quadratic matrix inequality. As a result of the negative sign in the $-P_{i} B_{i} R_{i}^{-1} B_{i}^{\mathrm{T}} P_{i}$ term, (7) cannot be simplified to an LMI. To accommodate the term $-P_{i} B_{i} R_{i}^{-1} B_{i}^{\mathrm{T}} P_{i}$, we introduce an additional design variable $X_{i}$. Since

$$
\left(X_{i}-P_{i}\right)^{\mathrm{T}} B_{i} R_{i}^{-1} B_{i}^{\mathrm{T}}\left(X_{i}-P_{i}\right) \geq 0
$$

for any $P_{i}$ and $X_{i}$ of the same dimensions, where the equality holds if $X_{i}=P_{i}$, we obtain a sufficient condition for the feasibility of inequality (7), given by

$$
\left[\begin{array}{cc}
\bar{Q}_{i}-P_{i} & A_{i}^{\mathrm{T}} P_{i} \\
P_{i} A_{i} & -P_{i}-\Theta\left(P_{i}, X_{i}\right)
\end{array}\right]+\Xi_{i}^{\mathrm{T}} \Xi_{i}<0
$$

where

$$
\begin{aligned}
\Theta\left(P_{i}, X_{i}\right)= & X_{i} B_{i} R_{i}^{-1} B_{i}^{\mathrm{T}} P_{i}+P_{i} B_{i} R_{i}^{-1} B_{i}^{\mathrm{T}} X_{i} \\
& -X_{i} B_{i} R_{i}^{-1} B_{i}^{\mathrm{T}} X_{i} .
\end{aligned}
$$

Theorem 1: There exists a solution $\left(P_{i}>0, F\right)$ satisfying matrix inequality (7) if and only if there exist matrices $F, P_{i}>0$, and $X_{i}>0$ satisfying matrix inequality (10) for $i=1, \ldots, r$.

This theorem can be established along a similar line as in [11].

Using Schur complement, inequality (10) is equivalent to the following matrix inequality

$$
\left[\begin{array}{ccc}
\bar{Q}_{i}-P_{i} & A_{i}^{\mathrm{T}} P_{i} & \left(F C_{i}\right)^{\mathrm{T}} R_{i}^{1 / 2} \\
P_{i} A_{i} & -P_{i}-\Theta\left(P_{i}, X_{i}\right) & P_{i} B_{i} R_{i}^{-1 / 2} \\
R_{i}^{1 / 2} F C_{i} & R_{i}^{-1 / 2} B_{i}^{\mathrm{T}} P_{i} & -I
\end{array}\right]<0 .
$$

This matrix inequality points to an iterative approach to solve for $F$ and $P_{i}$, namely, if $X_{i}$ is fixed in (11), then it reduces to an LMI problem on the unknown $F$ and $P_{i}$. The LMI problem is convex and can be solved if a feasible solution exists.

When $X_{i}$ is fixed, however, LMI (11) is only a sufficient condition for the feasibility of the matrix inequality (7). In fact, if we find a solution of LMI (11), then we find a solution of (7). But, in general, it has no solution for a fixed $X_{i}$. On the other hand, we can solve the optimal output feedback problem repeatedly for modified systems with the pair $\left(A_{i}, B_{i}, C_{i}\right)$ replaced by scaled pairs of the form $\left(A_{i} / \beta^{j}, B_{i} / \beta^{j}, C_{i}\right)$, where $\beta^{j} \geq 1$. This means that we can first determine a solution $F^{j}$ such that $\rho\left(A_{i}+B_{i} F^{j} C_{i}\right)<\beta^{j}$. That is, the closed-loop system matrices $A_{i}+B_{i} F^{j} C_{i}$ have eigenvalues in the $\beta^{j}$-circle of in the complex $z$-plane. From the above derivation, we 
obtain a necessary condition for the feasibility of (7). Thus, matrix inequality (7) has a solution $\left(P_{i}>0, F\right)$ then there exist a real number $\beta \geq 1$ and a matrix $X_{i}>0$ such that

$$
\left[\begin{array}{ccc}
\bar{Q}_{i}-P_{i} & A_{i}^{\mathrm{T}} P_{i} & \left(F C_{i}\right)^{\mathrm{T}} R_{i}^{1 / 2} \\
P_{i} A_{i} & -\beta^{2} P_{i}-P_{i} B_{i} R_{i}^{-1} B_{i}^{\mathrm{T}} P_{i} & P_{i} B_{i} R_{i}^{-1 / 2} \\
R_{i}^{1 / 2} F C_{i} & R_{i}^{-1 / 2} B_{i}^{\mathrm{T}} P_{i} & -I
\end{array}\right]<0
$$

Based on the idea that all eigenvalues of $A_{i}+B_{i} F C_{i}$ are reduced progressively toward the unit circle through the reduction of $\beta$, we may close in on the feasibility of (7). In other words, a stabilizing output gain will be found once $\beta \leq 1$. This technique can even be used to achieve a prescribed stability degree for the closed-loop system. The following algorithm is a discrete-time version of the algorithm proposed in [11].

Algorithm 1: ILMI Algorithm for LTI Discrete-Time Systems Step 1) SET $j=1, F^{j}=0$ and $\beta^{j}$ be a sufficiently large positive real number.

Step 2) LET $\hat{A}_{i}^{j}=\left(A_{i}+B_{i} F^{j} C_{i}\right) / \beta^{j}$ and SOLVE the following optimization problem for $P_{i}^{j}$.

OP1: Minimize $\sum_{i=1}^{r} \operatorname{trace}\left(P_{i}^{j}\right)$ subject to LMI constraints $P_{i}^{j}>0$ and

$$
\begin{gathered}
\left(\hat{A}_{i}^{j}\right)^{\mathrm{T}} P_{i}^{j} \hat{A}_{i}^{j}+\bar{Q}_{i}^{j}+\left(F^{j} C_{i}\right)^{\mathrm{T}} R_{i} F^{j} C_{i}-P_{i}^{j}<0, \\
i=1, \ldots, r
\end{gathered}
$$

where $\bar{Q}_{i}^{j}=Q_{i}+S_{i} F^{j} C_{i}+\left(S_{i} F^{j} C_{i}\right)^{\mathrm{T}}$. Set $X_{i}^{j}=P_{i}^{j}$.

Step 3) SOLVE the following optimization problem for $P_{i}^{j}, F^{j}$, and $\beta^{j}$.

OP2: Minimize $\beta^{j}$ subject to the LMI constraints:

$$
\left[\begin{array}{ccc}
\bar{Q}_{i}^{j}-P_{i}^{j} & A_{i}^{\mathrm{T}} P_{i}^{j} & * \\
P_{i}^{j} A_{i} & -\left(\beta^{j}\right)^{2} P_{i}^{j}-\Theta\left(P_{i}^{j}, X_{i}^{j}\right) & * \\
R_{i}^{1 / 2} F^{j} C_{i} & R_{i}^{-1 / 2} B_{i}^{\mathrm{T}} P_{i}^{j} & -I
\end{array}\right]<0
$$

for $i=1, \ldots, r$, where $*$ represents blocks that are readily inferred by symmetry and $\Theta\left(P_{i}^{j}, X_{i}^{j}\right)=X_{i}^{j} B_{i} R_{i}^{-1} B_{i}^{\mathrm{T}} P_{i}^{j}$ $+P_{i}^{j} B_{i} R_{i}^{-1} B_{i}^{\mathrm{T}} X_{i}^{j}-X_{i}^{j} B_{i} R_{i}^{-1} B_{i}^{\mathrm{T}} X_{i}^{j}$. Denote $\hat{\beta}^{j}$ and $\hat{F}^{j}$ as, respectively, the solutions $\beta^{j}$ and $F^{j}$ of OP2.

Step 4) IF $\hat{\beta}^{j} \leq 1$, SET $\beta^{j}=1$, GOTO Step 6, ELSE SET $j=j+1$ and $\bar{\beta}^{j}=\hat{\beta}^{j-1}, F^{j}=\hat{F}^{j-1}$.

Step 5) IF $\beta^{j-1}-\beta^{j}<\epsilon$, a pre-determined tolerance, GOTO Step 9, ELSE GOTO Step 2.

Step 6) SOLVE the following optimization problem for $P_{i}^{j}$ and $F^{j}$

OP3: Minimize $\sum_{i=1}^{r} \operatorname{trace}\left(P_{i}^{j}\right)$ subject to the LMI constraints.

$$
\left[\begin{array}{ccc}
\bar{Q}_{i}^{j}-P_{i}^{j} & A_{i}^{\mathrm{T}} P_{i}^{j} & * \\
P_{i}^{j} A_{i} & -P_{i}^{j}-\Theta\left(P_{i}^{j}, X_{i}^{j}\right) & * \\
R_{i}^{1 / 2} F^{j} C_{i} & R_{i}^{-1 / 2} B_{i}^{\mathrm{T}} P_{i}^{j} & -I
\end{array}\right]<0,
$$

for $i=1, \ldots, r$. Denote $\hat{P}_{i}^{j}$ and $\hat{F}^{j}$ as, respectively, the solutions $P_{i}^{j}$ and $F^{j}$ of OP3.

Step 7) IF $\sum_{i=1}^{r}\left\|\operatorname{trace}\left(X_{i}^{j}-\hat{P}_{i}^{j}\right)\right\|<\delta$, a pre-determined tolerance, GOTO Step 8, ELSE SET $j=j+1, X_{i}^{j}=\hat{P}_{i}^{j-1}$ GOTO Step 6.

Step 8) IF $\beta^{j}=1$, obtain the optimal solution $P_{i}^{\text {opt }}=\hat{P}_{i}^{j}$, $F^{\text {opt }}=\hat{F}^{j}$. STOP

Step 9) This algorithm cannot get a feasible solution. STOP.
Remark 2: In Step 1 , we may set $\beta^{1} \geq\|A\|$, then optimization OP1 must exist a feasible solution at $j=1$. For $j>1$, the existence of solution of OP1 can be guaranteed by (14) of the previous iteration. On the other hand, the existence of solution of optimization OP2 can be guaranteed by OP1. When a feasible solution is found in Step 3, that is, the solution of OP2 satisfies $\hat{\beta}^{j} \leq 1$, the algorithm will go to Step 6 to compute iteratively the optimal solution. At this step, optimization OP3 is feasible because OP2 has a solution $\hat{\beta}^{j} \leq 1$. For example, the solution of (14) $P_{i}^{j}$ and $F^{j}$ also satisfies (15). In the subsequent iterations, the solution of OP3 can be guaranteed by the solution of the previous iteration. If the initial conditions $x_{i}(0)$ of systems (1) are known, we should use the following minimization objective $\sum_{i=1}^{r}\left[x_{i}^{\mathrm{T}}(0) P_{i}^{j} x_{i}(0)\right]$ in OP1 and OP3 to compute the optimal feedback gain.

Remark 3: In practical control design, it is always desirable to design a control system which is not only stable, but also guarantees an adequate level of performance for every possible operating condition. Here, the guaranteed-cost performance design can also be treated and a minimization of a bound on all the performance measures, $\gamma$, can be sought where

$$
\operatorname{trace}\left(P_{i}\right) \leq \gamma, \quad i=1, \ldots, r
$$

since only the linear constraints $(16) \operatorname{trace}\left(P_{i}^{j}\right) \leq \gamma, i=1, \ldots, r$ are required to be added in OP1, OP2 and OP3.

Remark 4: The state feedback optimal design corresponds to the special case where $C_{i}=I$. With $Q_{i}=0$ and $R_{i}=I$, the proposed algorithm then determines the simultaneous stabilizability of $r$ plants via constant output feedback.

\section{Simultaneous LQ Design for MultiRate SAMPLED-DATA SYSTEMS}

To make a distinction, continuous-time signals will be represented by $(\cdot)$ around an independent variable, whereas discrete-time signals will be represented by bracket $[\cdot]$ in the following.

It is interesting to explore the possibility to simultaneously stabilize a collection of LTI systems using periodic time-varying output feedback gains. A trivial necessary condition that the optimal multi-model constant/static output feedback problem has a solution for a time-invariant multi-model system of the form (1) is that there exists a stabilizing output feedback gain which simultaneously stabilizes all the systems. It is a well known fact that not all systems are stabilizable by constant output feedback, thus the approach proposed in Section III is not generally applicable. However, analogous to the single model case we can try to employ periodic constant output feedback for the control of LTI systems which are not stabilizable via constant output feedback. From [2], [9], it is known that provided the period $N$ is chosen sufficiently high every LTI system can be stabilized by using periodic constant output feedback. Moreover, not only can each model of the multi-model system be stabilized by a periodic constant output feedback but an arbitrary fast dynamics can also be achieved simultaneously for all the models.

Consider the following LTI continuous-time systems:

$$
\begin{aligned}
\dot{x}_{i}(t) & =A_{i} x_{i}(t)+B_{i} u(t) \\
y_{i}(t) & =C_{i} x_{i}(t), \quad i=1, \ldots, r
\end{aligned}
$$

where $\left(A_{i}, B_{i}\right)$ and $\left(A_{i}, C_{i}\right)$ are assumed to be stabilizable and detectable, respectively. The objective is to design, if possible, a controller which performs well for each of these models. Assume that the sampling period is $h_{0}$, where $h_{0}$ is to be chosen such that $\left(e^{A_{i} h_{0}}, C_{i}\right)$ is detectable for $i=1, \ldots, r$. The output measurements are taken at 
time instants $t=k h_{0}, k=0,1,2, \ldots$. The periodic output feedback control law is given by

$$
u(t)=F(t) y\left(k h_{0}\right), \quad F\left(t+h_{0}\right)=F(t),
$$

for $k h_{0} \leq t<(k+1) h_{0}$, which simultaneously minimizes the following continuous-time performance index:

$$
J_{i}=\mathcal{E}\left\{\int_{0}^{\infty}\left[\begin{array}{l}
x_{i}(t) \\
u_{i}(t)
\end{array}\right]\left[\begin{array}{cc}
Q_{i} & 0 \\
0 & R_{i}
\end{array}\right]\left[\begin{array}{l}
x_{i}(t) \\
u_{i}(t)
\end{array}\right] d t\right\}
$$

for $i=1, \ldots, r$.

We consider a controller which samples all the plant outputs with the same period $h_{0}$, and changes the $i$ th plant input with shorter pe$\operatorname{riod} h_{i}$ (and is kept constant over the time interval of $h_{i}$ ), where $h_{i}=$ $h_{0} / N_{i}$, and $N_{i}$ are some positive integers. $h_{0}$ is called frame period, and $N_{i}$ input multiplicity. This means that each component is fed into the system by means of the following zeroth-order hold mechanism

$$
u_{i}(t)=u_{i}\left(j h_{i}\right), \quad t \in\left[j h_{i},(j+1) h_{i}\right) .
$$

As in [2] and [8], the control law is assumed to be piecewise constant. That is, it is given by

$$
u_{i}\left(k h_{0}+j h_{i}\right)=f_{i j}(k) y\left(k h_{0}\right)
$$

for $i=1, \ldots, m, j=0, \ldots, N_{i}-1$, or

$$
u_{i}[k N+j]=f_{i j}(k) y[k N]
$$

where $f_{i j}(k)$ is a row vector denoting the time-varying feedback gain. This type of controller is called multirate input constant output feedback controller [2], [8], or piecewise constant output feedback controller.

\section{A. Piecewise Constant Output Feedback System}

For simplicity, we first consider the following single LTI continuous-time system

$$
\dot{x}(t)=A x(t)+B u(t), \quad y(t)=C x(t) .
$$

Let $N$ be the least-common multiple of the entries of $\left\{N_{i} \mid i=1, \ldots, m\right\}$ and $h=h_{0} / N, n_{i}=N / N_{i}$. Let $\left(A_{d}, B_{d}, C\right)$ denote the discrete-time system of (22) at rate $1 / h$. That is, $A_{d}=\exp (A h), B_{d}=\int_{0}^{h} \exp (A s) B d s$. The discretized system of (22) at rate $1 / h$ is as follows:

$$
x[l+1]=A_{d} x[l]+B_{d} u[l], \quad y[l]=C x[l] .
$$

Denote $\bar{b}_{i}=\int_{0}^{h_{i}} \exp (A s) b_{i} d s$, where $b_{i}$ is the $i$ th column of $B$. Define

$$
\begin{aligned}
& \bar{A}=A_{d}^{N}, \\
& \bar{B}=\left[\begin{array}{lllll}
A_{d}^{n_{1}\left(N_{1}-1\right)} \bar{b}_{1} & \cdots & \bar{b}_{1} & \cdots
\end{array}\right. \\
& \left.A_{d}^{n_{m}\left(N_{m}-1\right)} \bar{b}_{m} \quad \cdots \quad \bar{b}_{m}\right] \in \mathcal{R}^{n \times \bar{N}} \\
& \bar{F}_{k}=\left[\begin{array}{lll}
\hat{f}_{1}^{\mathrm{T}}(k) & \cdots & \hat{f}_{m}^{\mathrm{T}}(k)
\end{array}\right] \in \mathcal{R}^{\bar{N} \times p}
\end{aligned}
$$

where

$$
\bar{N}=\sum_{i=1}^{m} N_{i}, \quad \hat{f}_{i}(k)=\left[\begin{array}{lll}
f_{i, 0}^{\mathrm{T}}(k) & \cdots & f_{i, N_{i}-1}^{\mathrm{T}}(k)
\end{array}\right]^{\mathrm{T}} .
$$

Define

$$
\bar{B}(j)=\left[\begin{array}{lll}
\hat{b}_{1}(j) & \cdots & \hat{b}_{m}(j)
\end{array}\right]
$$

where $\hat{b}_{i}(j) \in \mathcal{R}^{n \times N_{i}}, j=0, \ldots, N$ are defined as

$$
\begin{aligned}
& \hat{b}_{i}(0)=0 \\
& \hat{b}_{i}\left(v_{i}\right)=\left[\begin{array}{llll}
\check{b}_{i}\left(v_{i}\right) & 0 & \cdots & 0
\end{array}\right] \\
& \hat{b}_{i}\left(n_{i}+v_{i}\right)=\left[\begin{array}{lllll}
A_{d}^{v} \bar{b}_{i} & \check{b}_{i}\left(v_{i}\right) & 0 & \cdots & 0
\end{array}\right], \\
& \hat{b}_{i}\left(\left(N_{i}-1\right) n_{i}+v_{i}\right)=\left[\begin{array}{lll}
A_{d}^{\left(N_{i}-2\right) n_{i}+v_{i}} \bar{b}_{i} & \cdots
\end{array}\right. \\
& \left.A_{d}^{n_{i}+v_{i}} \bar{b}_{i} \quad A_{d}^{v_{i}} \bar{b}_{i} \check{b}_{i}\left(v_{i}\right)\right]
\end{aligned}
$$

where $\check{b}_{i}\left(v_{i}\right)=\int_{0}^{v_{i} h} \exp (A s) b_{i} d s, v_{i}=1, \ldots, n_{i}$. Obviously,

$$
\begin{array}{ll}
\check{b}_{i}\left(n_{i}\right)=\bar{b}_{i}, & \hat{b}_{i}(N)=\left[\begin{array}{lll}
A_{d}^{\left(N_{i}-1\right) n_{i}} \bar{b}_{i} & \cdots & \bar{b}_{i}
\end{array}\right] \\
\bar{B}(N)=\bar{B}, & \bar{B}(j) \in \mathcal{R}^{m \times \bar{N}} .
\end{array}
$$

From (22), we have

$$
\begin{aligned}
& x\left(k h_{0}+j h\right) \\
& \quad=A_{d}^{j} x\left(k h_{0}\right)+\int_{k h_{0}}^{k h_{0}+j h} \exp \left[A\left(k h_{0}+j h-t\right)\right] B u(t) d t \\
& \quad=A_{d}^{j} x\left(k h_{0}\right)+V_{k}(j) y\left(k h_{0}\right)
\end{aligned}
$$

where

$$
\begin{aligned}
V_{k}(j) & =\int_{k h_{0}}^{k h_{0}+j h} \exp \left[A\left(k h_{0}+j h-t\right)\right] B F(t) d t \\
& =\int_{k h_{0}}^{k h_{0}+j h} \exp \left[A\left(k h_{0}+j h-t\right)\right] \sum_{i=1}^{m} b_{i} f_{i}(t) d t \\
& =\sum_{i=1}^{m} V_{k}(j, i)
\end{aligned}
$$

where $f_{i}(t)$ is the $i$ th row vector of $F(t)$ and

$$
\begin{aligned}
V_{k}(j, i) & \\
= & \int_{k h_{0}}^{k h_{0}+j h} \exp \left[A\left(k h_{0}+j h-t\right)\right] b_{i} f_{i}(t) d t \\
= & \sum_{l=0}^{\bar{n}_{i j}-1} \int_{k h_{0}+l h_{i}}^{k h_{0}+(l+1) h_{i}} \exp \left[A\left(k h_{0}+j h-t\right)\right] b_{i} f_{i l}(k) d t \\
& +\int_{k h_{0}+\bar{n}_{i j} h_{i}}^{k h_{0}+j h} \exp \left[A\left(k h_{0}+j h-t\right)\right] b_{i} f_{i, \bar{n}_{i j}}(k) d t
\end{aligned}
$$

where $\bar{n}_{i j}$ is an integer satisfying $\bar{n}_{i j} n_{i} \leq j<\left(\bar{n}_{i j}+1\right) n_{i}$. Obviously $0 \leq \bar{n}_{i j} \leq N_{i}$ for all $j$. Note that $f_{i l}(k)$ is a constant vector. Hence,

$$
\begin{aligned}
V_{k}(j, i)= & \sum_{l=0}^{\bar{n}_{i j}-1} \int_{l h_{i}}^{(l+1) h_{i}} \exp [A(j h-t)] b_{i} d t f_{i l}(k) \\
& +\int_{\bar{n}_{i j} h_{i}}^{j h} \exp [A(j h-t)] b_{i} d t f_{i, \bar{n}_{i j}}(k) \\
= & \sum_{l=0}^{\bar{n}_{i j}-1} \exp \left[A\left(\left(\bar{n}_{i j}-l-1\right) n_{i}+v_{i}\right) h\right] \\
& \cdot \int_{0}^{h_{i}} \exp \left[A\left(h_{i}-t\right)\right] b_{i} d t f_{i l}(k) \\
& +\int_{0}^{v_{i} h} \exp \left[A\left(v_{i} h-t\right)\right] b_{i} d t f_{i, \bar{n}_{i j}}(k) \\
= & \hat{b}_{i}(j) \hat{f}_{i}(k)
\end{aligned}
$$


where $v_{i}=j-\bar{n}_{i j} n_{i}$. Hence

$$
\begin{aligned}
x\left(k h_{0}+j h\right) & =A_{d}^{j} x\left(k h_{0}\right)+\sum_{i=1}^{m} \hat{b}_{i}(j) \hat{f}_{i}(k) y\left(k h_{0}\right) \\
& =A_{d}^{j} x\left(k h_{0}\right)+\bar{B}(j) \bar{u}\left(k h_{0}\right) \\
\bar{u}\left(k h_{0}\right) & =\bar{F}_{k} y\left(k h_{0}\right)
\end{aligned}
$$

where $\bar{u}(t)$ is an augmented input and maintains constant on the interval $\left[k h_{0},(k+1) h_{0}\right)$. The discrete formula of above equations are

$$
\begin{aligned}
x[k N+j] & =A_{d}^{j} x[k N]+\bar{B}(j) \bar{u}[k N] \\
\bar{u}[k N] & =\bar{F}_{k} y(k N), \quad j=0, \ldots, N
\end{aligned}
$$

where $\bar{u}[k N+j]$ will maintain constant for $j=0, \ldots, N-1$. Then, we have

$$
x[(k+1) N]=\bar{A} x[k N]+\bar{B} \bar{u}[k N]
$$

which is the discretized state equation of continuous-time system (22) with sampling time $h_{0}$. With (27), we can easily compute the discretized state response with sampling time $h$.

Remark 5: If the multirate controller (20) is assumed to be periodic piecewise constant, the transition matrix (for the frame period $h_{0}$ ) of the closed-loop system becomes $\Phi=\bar{A}+\overline{B F} C$ (see [2]) where

$$
\bar{F}=\left[\begin{array}{lllllll}
f_{1,0}^{\mathrm{T}} & \cdots & f_{1, N_{1}-1}^{\mathrm{T}} & \cdots & f_{m, 0}^{\mathrm{T}} & \cdots & f_{m, N_{m}-1}^{\mathrm{T}}
\end{array}\right]^{\mathrm{T}}
$$

is a constant matrix. From (29), we can easily obtain the above transition matrix. It is shown in [2] that for almost every $h_{0}$, the matrix $\overline{B F}$ can be assigned an arbitrary value by suitable choice of $\bar{F}$ if $(A, B)$ is controllable and if $N_{i} \geq v_{i}, i=1, \ldots, m$, where $v_{i}$ is a locally minimum controllability index of $(A, B)$ because $\bar{B}$ has rank $n$ in such case. If $(C, A)$ is observable, then the eigenvalues of $\Phi$ can be assigned arbitrarily for almost every $h_{0}$ by suitable choice of $\bar{F}$.

\section{B. Discretization of Quadratic Performance Index}

Consider the continuous-time quadratic performance index

$$
\min _{u[k]}\left\{J=\mathcal{E}\left\{\int_{0}^{\infty}\left[x^{\mathrm{T}}(t) Q x(t)+u^{\mathrm{T}}(t) R u(t)\right] d t\right\}\right\}
$$

where $R>0$ and $Q \geq 0$ are constant matrices. Sampling (30) at rate $1 / h$ yields the equivalent discrete-time weights [1]

$$
\begin{aligned}
R_{d} & =\int_{0}^{h}\left(\bar{H}^{\mathrm{T}}(t) Q \bar{H}(t)+R\right) d t \\
Q_{d} & =\int_{0}^{h}\left(e^{A t}\right)^{\mathrm{T}} Q e^{A t} d t, \quad S_{d}=\int_{0}^{h}\left(e^{A t}\right)^{\mathrm{T}} Q \bar{H}(t) d t
\end{aligned}
$$

where $\bar{H}(t)=\int_{0}^{t} e^{A s} B d s$. It can be proven that the matrix $Q_{d}-$ $S_{d} R_{d}^{-1} S_{d}^{\mathrm{T}} \geq 0$ if $Q \geq 0$ and $R>0$, and $Q_{d}-S_{d} R_{d}^{-1} S_{d}^{\mathrm{T}}>0$ if $Q>0$ and $R>0$.

Then the sampled-data optimal control problem becomes finding multirate feedback control law (20) to minimize the following discrete-time performance index

$$
J=\mathcal{E}\left\{\sum_{j=0}^{\infty}\left[\begin{array}{l}
x[l] \\
u[l]
\end{array}\right]^{\mathrm{T}}\left[\begin{array}{ll}
Q_{d} & S_{d} \\
S_{d}^{\mathrm{T}} & R_{d}
\end{array}\right]\left[\begin{array}{l}
x[l] \\
u[l]
\end{array}\right]\right\}
$$

with the piecewise constant output feedback $u[l]$ at rate $1 / h$. From the last subsection, we can rewrite (29) as a LTI system

$$
\begin{aligned}
x_{N}[k+1] & =\bar{A} x_{N}[k]+\bar{B} u_{N}[k] \\
y_{N}[k] & =C x_{N}[k] \\
u_{N}[k] & =\bar{F}_{k} y_{N}[k]
\end{aligned}
$$

which can be seen as the sampling of system (23) at $N$, where $x_{N}[k]=$ $x[k N], y_{N}[k]=y[k N], u_{N}[k]=\bar{u}[k N], k \geq 0$, with initial condition $x_{N}[0]=x[0]$. The input $u[k N+j]$ in (23) and (31) can be written as

$$
\begin{gathered}
u[k N+j]=F_{j}(k) y[k N]=W_{j+1} u_{N}[k] \\
j=0, \ldots, N-1
\end{gathered}
$$

where $W_{j}=\left\{w_{i l}^{j}\right\} \in \mathcal{R}^{m \times \bar{N}}$, are defined as

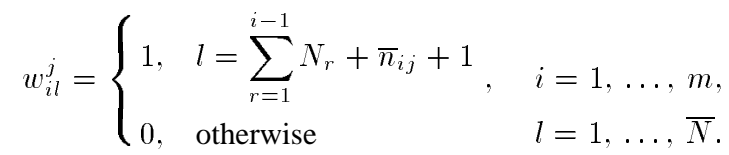

The LTI system (32) can be seen as a state-sampled representation of (23), feeding by an augmented input vector (34) and producing an augmented output vector (33). It is easy to find that system (32) is stable if and only if system (23) is asymptotically stable. Consequently, with initial condition $x_{N}[0]=x[0]$, (31) can be rewritten as

$$
J=\mathcal{E}\left\{\sum_{k=0}^{\infty}\left[\begin{array}{l}
x_{N}[k] \\
u_{N}[k]
\end{array}\right]^{\mathrm{T}}\left[\begin{array}{cc}
\tilde{Q} & \tilde{S} \\
\tilde{S}^{\mathrm{T}} & \tilde{R}
\end{array}\right]\left[\begin{array}{l}
x_{N}[k] \\
u_{N}[k]
\end{array}\right]\right\}
$$

where $\tilde{Q}, \tilde{S}$ and $\tilde{R}$ are time-invariant matrices

$$
\begin{aligned}
\tilde{Q}= & \sum_{j=0}^{N-1}\left(A_{d}^{j}\right)^{\mathrm{T}} Q_{d} A_{d}^{j} \\
\tilde{R}= & \sum_{j=0}^{N-1} W_{j+1}^{\mathrm{T}} R_{d} W_{j+1}+\sum_{j=1}^{N-1} \\
& \cdot\left(\bar{B}(j)^{\mathrm{T}} Q_{d} \bar{B}(j)+\bar{B}(j)^{\mathrm{T}} S_{d} W_{j+1}+W_{j+1}^{\mathrm{T}} S_{d}^{\mathrm{T}} \bar{B}(j)\right) \\
\tilde{S}= & \sum_{j=0}^{N-1}\left(A_{d}^{j}\right)^{\mathrm{T}} S_{d} W_{j+1}+\sum_{j=1}^{N-1}\left(A_{d}^{j}\right)^{\mathrm{T}} Q_{d} \bar{B}(j) .
\end{aligned}
$$

The above derivation leads to the following result.

Theorem 2: Assume that continuous-time system (22) is given. The optimal design of periodic constant output feedback control law (20) minimizing continuous-time performance (30) can be reduced to that of a constant output feedback control law $\bar{F}$ minimizing the discrete-time performance index (37).

\section{Simultaneous LQ Optimal Design}

The above derivation for a single plant can be easily extended to simultaneous LQ optimal design for $r$ continuous-time systems (17) by the same periodic constant output feedback controller (20). By the derivation of the last subsection, the continuous-time system model (17) and the periodic piecewise constant output feedback control law (20) can be reduced to the following LTI discrete-time system models:

$$
\begin{aligned}
x_{i, N}[k+1] & =\bar{A}_{i} x_{i, N}[k]+\bar{B}_{i} u_{i, N}[k] \\
y_{i, N}[k] & =C_{i} x_{i, N}[k] \\
u_{i, N}[k] & =\bar{F} y_{i, N}[k]
\end{aligned}
$$

where $\bar{A}_{i}, \bar{B}_{i}$ can be determined similarly as in (24) and (25), respectively.

Theorem 3: Assume that the $r$ continuous-time systems (17) are given. The simultaneous optimal design of periodic constant output feedback control law (20) minimizing continuous-time performances 
TABLE I

COMPARISON OF RESULTS AT DIFFERENT SAMPLING RATES

\begin{tabular}{l|l|l|l}
\hline$h$ & $N$ & $J$ & $F$ \\
\hline 1 & 1 & 6.648 & 0.5089 \\
\hline & 3 & 4.619 & $-0.6259,0.4218,1.2677$ \\
\hline & 5 & 4.512 & $-0.8561,-0.1809,0.4161,0.9407,1.4005$ \\
\hline & 10 & 4.468 & $\begin{array}{l}-1.035,-0.6785,-0.3421,-0.0255,0.2718 \\
0.5507,0.8119,1.0566,1.2856,1.4998\end{array}$ \\
\hline & & & \\
\hline 3 & 1 & 28.28 & 0.1635 \\
\hline & 3 & 23.22 & $1.0602,0.4401,-0.0381$ \\
\hline & 5 & 23.26 & $1.1241,0.8494,0.4409,0.1130,-0.0677$ \\
\hline & 10 & 23.27 & $\begin{array}{l}1.1385,1.0875,0.9410,0.7464,0.5401 \\
\end{array}$ \\
\hline 5 & 1 & 9.305 & $0.3479,0.1851,0.0591,-0.0296,-0.0847$ \\
\hline & 3 & 4.445 & $0.2031,0.6426,-0.1489$ \\
\hline & 5 & 3.932 & $-0.1322,0.7263,0.4478,0.0625,-0.1314$ \\
\hline & 10 & 3.756 & $-0.4978,0.2996,0.6333,0.6483,0.4987$ \\
& & & $0.303,0.1294,0.0036,-0.0792,-0.1356$ \\
\hline 10 & 1 & 12.73 & 0.3864 \\
\hline & 3 & 9.998 & $0.1384,-0.1507,0.1382$ \\
\hline & 5 & 3.560 & $0.1916,0.6673,-0.3037,0.0687,0.0048$ \\
\hline & 10 & 2.310 & $-0.5429,0.8156,0.5861,0.1015,-0.1004$ \\
& & & $-0.0814,-0.0173,0.0131,0.0125,0.0016$ \\
\hline
\end{tabular}

(18) can be reduced to that of a constant output feedback control law $\bar{F}$ minimizing the discrete-time performance index

$$
J_{i}=\mathcal{E}\left\{\sum_{k=0}^{\infty}\left[\begin{array}{l}
x_{i, N}[k] \\
u_{i, N}[k]
\end{array}\right]^{\mathrm{T}}\left[\begin{array}{cc}
\tilde{Q}_{i} & \tilde{S}_{i} \\
\tilde{S}_{i}^{\mathrm{T}} & \tilde{R}_{i}
\end{array}\right]\left[\begin{array}{l}
x_{i, N}[k] \\
u_{i, N}[k]
\end{array}\right]\right\}
$$

for the LTI discrete-time systems (41), (42), where $\tilde{Q}_{i}, \tilde{R}_{i}$, and $\tilde{S}_{i}$ can be determined similarly as in (38)-(40), respectively, for $i=1, \ldots, r$.

Based on the above theorem, after selecting the frame period $h_{0}$ and the input multiplicities $N_{i}$, Algorithm 1 of Section III can be used to solve the feedback gain $\bar{F}$.

\section{EXAMPLES}

Example 1: Consider the following harmonic oscillator model:

$$
\begin{aligned}
& \dot{x}(t)=\left[\begin{array}{rr}
0 & 1 \\
-1 & 0
\end{array}\right] x(t)+\left[\begin{array}{l}
0 \\
1
\end{array}\right] u(t) \\
& y(t)=\left[\begin{array}{ll}
1 & 0
\end{array}\right] x(t), \quad x_{0}(t)=\left[\begin{array}{ll}
1 & 1
\end{array}\right]^{\mathrm{T}}
\end{aligned}
$$

and we wish to minimize the linear quadratic regulator index

$$
J=\int_{0}^{\infty} \frac{1}{2}\left[x_{1}^{2}(t)+x_{2}^{2}(t)+u^{2}(t)\right] d t
$$

Table I summarizes the resulting discrete performances at different sampling rates using periodic constant output feedback obtained by our ILMI algorithm. We find that as $N$ increases, the optimal performance index decreases, when $N$ is much larger than the system controllability index (in this example, it is equal to Step 1, the optimal performance indexes at different rates are close to that of continuous periodic output feedback given in [7]. However, when $h=3$ and $h=10, N=3$, weak performance indexes are obtained. This is because both cases are simply too close to the inadmissible sampling time $\pi$ [1].

Example 2: Consider two plants

$$
G_{1}(s)=\frac{1}{s+1}, \quad G_{2}(s)=\frac{a s+1}{(s+1)(s-1)}
$$

where $a \neq 1$ is a constant. It is not difficult to deduce that when $a<-1$, the plants are not simultaneously stabilizable by a linear time-invariant controller. Now we use the approach of the last section
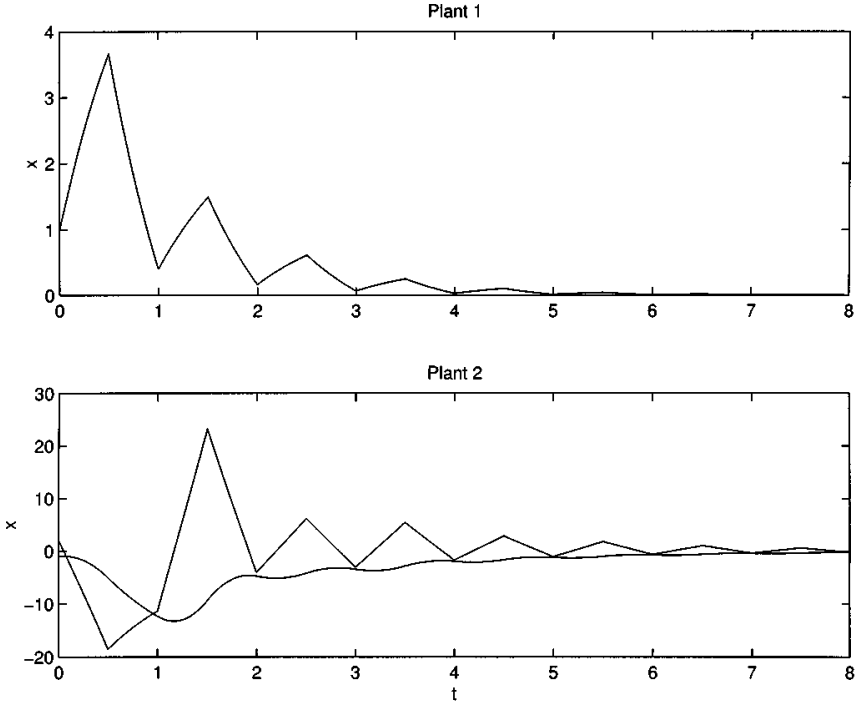

Fig. 1. State responses with periodic constant controller $\bar{F}^{1}$.
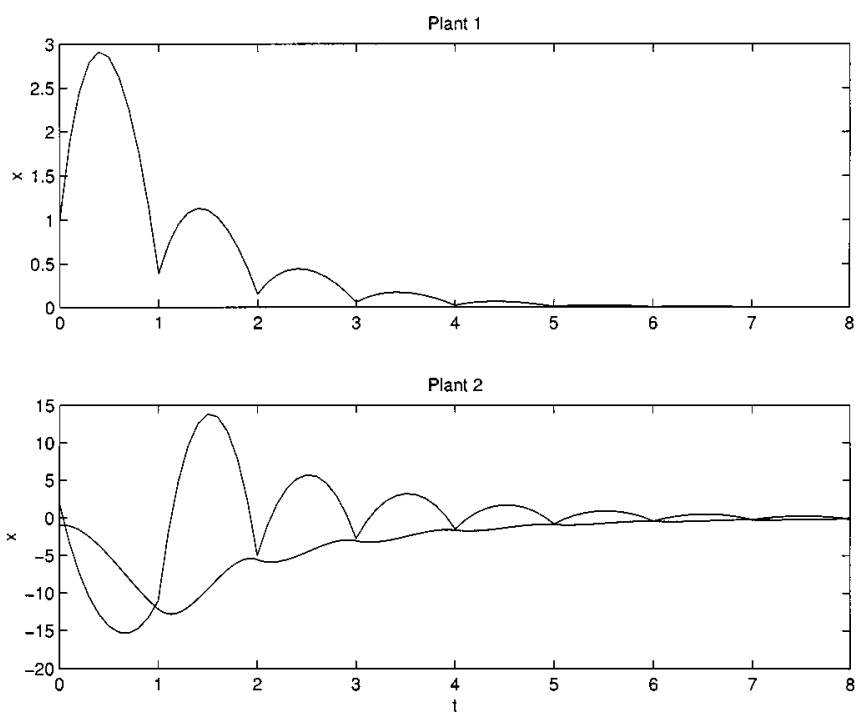

Fig. 2. State responses with periodic constant controller $\bar{F}^{2}$.

to find a simultaneous stabilizing periodic controller for $a=-2$. That is, we take $Q_{i}=0.001 I$ and $R_{i}=0.001, S_{i}=0$ for $i=1,2$. Let the sampling time be $h=1$. When $N=1$, the ILMI algorithm cannot obtain a feasible solution, but when we select $N=2$, after 25 iterations we obtain

$$
\bar{F}^{1}=\left[\begin{array}{ll}
7.783 & -4.682
\end{array}\right]^{\mathrm{T}} .
$$

This means that the above two plants can be simultaneously stabilized by a 2-periodic piecewise output feedback gain. When we set $N=10$, after 21 iterations we obtain control law

$$
\begin{gathered}
\bar{F}^{2}=\left[\begin{array}{cccccc}
10.161 & 7.992 & 5.984 & 4.096 & 2.290 & 0.5304 \\
-1.219 & -2.993 & -4.827 & -6.757
\end{array}\right]^{\mathrm{T}} .
\end{gathered}
$$

Figs. 1 and 2 show state responses of the plants with the above periodic piecewise constant controllers under initial conditions $x_{0}^{1}=1$ and $x_{0}^{2}=[2,-1]^{\mathrm{T}}$. Obviously, the states of both systems converge to 0 . However, the curves are much smoother when $N$ is bigger. On the other hand, although the systems are stabilized by setting $N=2$, the systems have larger overshoot when $N$ is small. 


\section{REFERENCES}

[1] H. Al-Rahmani and G. F. Franklin, "A new optimal multirate control of linear periodic and time-invariant systems," IEEE Trans. Automat. Contr., vol. 35, pp. 406-415, Apr. 1990.

[2] M. Araki and T. Hagiwara, "Pole assignment by multirate sampled-data output feedback," Int. J. Contr., vol. 44, pp. 1661-1673, 1986.

[3] S. Boyd, L. El Ghaoui, E. Feron, and V. Balakrishnan, Linear Matrix Inequalities in System and Control Theory. Philadelphia, PA: SIAM, 1994.

[4] Y.-Y. Cao and Y.-X. Sun, "Static output feedback simultaneous stabilization: ILMI approach,” Int. J. Contr., vol. 70, no. 5, pp. 803-814, 1998.

[5] Y.-Y. Cao, Y.-X. Sun, and J. Lam, "Simultaneous stabilization via static output feedback and state feedback," IEEE Trans. Automat. Contr., vol. 44, pp. 1277-1282, June 1999.

[6] A. Feuer and C. A. Goodwin, "Generalized sampled-data functions: Frequency domain analysis of robustness, sensitivity, and intersample difficulties," IEEE Trans. Automat. Contr., vol. 39, pp. 1042-1047, May 1994.

[7] G. L. Hyslop, H. Schattler, and T.-J. Tarn, "Descent algorithms for optimal periodic output feedback control," IEEE Trans. Automat. Contr. vol. 37, pp. 1893-1904, Dec. 1992.

[8] P. T. Kabamba, "Control of linear systems using generalized sampled-data hold functions," IEEE Trans. Automat. Contr., vol. AC-32, pp. 772-783, Sept. 1987.

[9] P. P. Khargonekar, K. Poolla, and A. Tannenbaum, "Robust control of linear time invariant plants using periodic compensation," IEEE Trans. Automat. Contr., vol. AC-30, pp. 1088-1096, Nov. 1985.

[10] V. Kučera and C. de Souza, "A necessary and sufficient condition for output feedback stabilizability," Automatica, vol. 31, no. 9, pp. $1357-1359,1995$

[11] J. Lam and Y.-Y. Cao, "Simultaneous linear-quadratic optimal control design via static output feedback," Int. J. Robust Nonlin. Contr., vol. 9, no. 9, pp. 551-558, 1999

[12] M. Paskota, V. Sreeram, K. L. Teo, and A. I. Mees, "Optimal simultaneous stabilization of linear single-input systems via linear state feedback," Int. J. Contr., vol. 60, no. 4, pp. 483-498, 1994.

\section{Attributed Concept Maps: Fuzzy Integration and Fuzzy Matching}

\author{
S.-W. Chen, S. C. Lin, and K. E. Chang
}

Abstract-A concept map, typically depicted as a connected graph, is composed of a collection of propositions. Each proposition forming a semantic unit consists of a small set of concept nodes interconnected to one another with relation links. Concept maps possess a number of appealing features which make them a promising tool for teaching, learning, evaluation, and curriculum planning. In this paper, we extend concept maps by associating their concept nodes and relation links with attribute values which indicate the relative significance of concepts and relationships in knowledge representation. The resulting maps are called attributed concept maps (ACM). Assessing students will be conducted by matching their ACMs with those prebuilt by experts. The associated techniques are referred to as map matching techniques. The building of an expert ACM has in the past been done by only one specialist. In this study, we integrate a number of maps developed by separate experts into a single map, called the master map (MM), which will serve as a prototypical map in map matching. Both map integration and map matching are conceptualized in terms of fuzzy set discipline. Experimental results have shown that the proposed ideas of ACM, MM, fuzzy map integration, and fuzzy map matching are well suited for students with high performances and difficult subject materials.

Index Terms-Attributed concept maps (ACMs), concept mapping, fuzzy map integration, fuzzy map matching, master maps (MMs).

\section{NOMENCLATURE}

CM Concept map.

$\mathrm{ACM}$

EACM

$\mathrm{MM}$

$X$

$A, B$

$g()$

$g_{\lambda}()$

$\lambda$

$d_{i}$

$S=\left\{s_{1}, \ldots, s_{n}\right\}$

$S^{\prime}=\left\{s_{1}^{\prime}, \ldots, s_{n}^{\prime}\right\}$

$M_{i}$

$M_{i}^{\prime}$

$N_{i}, L_{i}$

Attributed concept map.

Extended attributed concept map.

Master map.

Universal set.

Subsets of $X$.

Fuzzy measure function.

Sugeon fuzzy measure function.

Sugeon fuzzy measure parameter.

Fuzzy density of $x_{i} \in X$.

Set of specialists.

Sorted set of specialists.

$i$ th ACM.

$i$ th EACM.

Sets of nodes and links of ACM $M_{i}$, respectively.

e

$a_{i}$

$a_{i j}$ Entity representing a node or a link.

Attribute value of the $i$ th node.

Attribute value of the link connecting the $i$ th node and $j$ th node.

$C\left(a_{j}\right) \quad$ Confidence of attribute $a_{i}$.

$c_{i}\left(s_{j}\right) \quad$ Confidence of specialist $s_{j}$ in attribute $a_{i}$.

$\alpha \quad$ Positive constant.

$H^{(i)} \quad$ Set of first-order neighbors of the $i$ th node.

$\tilde{H}^{(i)} \quad$ Fuzzy version of $H^{(i)}$.

$\mu_{\tilde{H}}^{(i)} \quad$ Membership function of $\tilde{H}^{(i)}$.
Manuscript received January 1, 2000; revised March 27, 2001. This work was supported by the National Science Council, Taiwan, R.O.C., under Contract NSC-88-2520-S-003-003. This paper was recommended by Associate Editor A. Kandel.

The authors are with the Department of Information and Computer Education, National Taiwan Normal University, Taipei, Taiwan, R.O.C. (e-mail: schen@ice.ntnu.edu.tw).

Publisher Item Identifier S 1083-4419(01)05976-3. 